\title{
Juguetes como instrumentos de enseñanza en ingeniería: El caso del pájaro bebedor
}

CHEMICAL ENGINEERING

\section{Toys as teaching tools in engineering: The case of drinking bird}

\author{
Simón Reif-Acherman ${ }^{\S}$ \\ Escuela de Ingeniería Química, Universidad del Valle, Cali, Colombia \\ §simon.reif@correounivalle.edu.co
}

(Recibido: Septiembre 5 de 2012 - Aceptado: Abril 1 de 2013)

\begin{abstract}
Resumen
Los procesos de enseñanza en ingeniería han venido evolucionando desde la llamada clase magistral, con un formato casi que inflexible y que en buena proporción aun hoy en día presentan los conceptos y principios de manera abstracta y sin relación con la realidad que el estudiante conoce, a un esquema más pedagógico que permite una mayor conexión con el entorno. Un muy bien conocido y representativo juguete se usa en este artículo para mostrar como ejemplos relacionados de la vida real que rodean la vida de los estudiantes pueden usarse para complementar los conceptos teóricos, en este caso físicos, y desarrollos matemáticos que rodean un tema específico, contribuyendo así positivamente a la motivación para el estudio y análisis de sistemas más complejos.
\end{abstract}

Palabras clave: Aplicaciones reales, eficiencia pedagógica, enseñanza en ingeniería, modelos y juguetes, motivación, termodinámica

\begin{abstract}
Learning processes in engineering had been evolving from the so-called master class, with an almost inflexible format and that presented, still nowadays in great proportion, concepts and principles in an abstract way and without relation to the reality the student knows, to a more pedagogical scheme which allows greatest connection with the environment. One well-known and representative toy is used in this article to show how real-life related examples surrounding the students' world can be used in order to complement theoretical concepts, in this case physical, and mathematical developments around a specific subject, positively contributing so to the motivation for the studying and analysis of more complex systems.
\end{abstract}

Keywords: Engineering education, Models and toys, Motivation, Pedagogical efficiency, Real applications, Thermodynamics 


\section{Introducción}

"No me CUENTE, DEMUESTRELO!!!".

Uno de los problemas más comunes y difíciles de resolver en la enseñanza de la ciencia y la ingeniería es el de lograr desarrollar entre los estudiantes un sentido de aplicación de los principios científicos de cualquier tipo a situaciones prácticas de la vida diaria. La dificultad para entender ciertos conceptos abstractos presentes en las presentaciones iniciales de numerosos tópicos en ingeniería hace que el trabajo del estudiante se limite frecuentemente a la simple y automática memorización de un casi interminable número de ecuaciones, métodos y constantes numéricas, así como a la realización mecánica, prácticamente "robótica", de las prácticas de laboratorio correspondientes. Es en ese momento cuando frases como "¿eso tan complejo si sirve para algo?”, "¿hay alguien a quien le guste este tema?", o similares, empiezan a oírse en los corredores de la institución.

Las demostraciones visuales de fenómenos relacionados con dichos conceptos permiten consolidar el establecimiento de una relación entre las actividades académicas teóricas y el mundo real exterior. De no existir dichas demostraciones, esa relación difícilmente rebasa las fronteras del salón de clase, y, de hacerlo, generalmente concluye con la presentación de los exámenes respectivos.

Pedagógicamente se ha comprobado que esas demostraciones sirven de puente entre el instintivo conocimiento natural de los estudiantes y la teoría, despiertan su entusiasmo, los mantiene activos, y facilita el aprendizaje. Escuelas, colegios y universidades han venido incrementando el uso de instrumentos didácticos con propósitos pedagógicos para demostrar conocidas leyes físicas, evaluar, en aquellos casos donde resulte posible, el efecto de diversas variables a una escala diferente del prototipo original reproducido, y elaborar y mostrar diversos fenómenos de una manera más próxima a la realidad. Las diversas clases de instrumentos existentes incluyen elementos capaces de realizar simulacros mecánicos de fenómenos naturales, otros que reflejan descubrimientos accidentales, algunos involucrando nuevas técnicas o materiales, etc.. Estos instrumentos pueden ser 'juguetes', capaces de producir entretenimiento, sorpresas asociadas a principios físicos simples, o efectos aparentemente paradójicos, o 'modelos' a pequeña escala de algún elemento real cuyo nivel de representación depende de la respectiva aplicación. Debido al contexto en el que se relacionan en el presente artículo, los términos juguete y modelo serán usados aquí indistintamente.

Colectivamente los juguetes representan el resultado de siglos de observación y análisis científicos. Supapelhavenidoevolucionandodesde su aparición como mero objeto de entretención (Al-Gailani, 2009; Breni, 2012; Turner, 1992), hasta convertirse en la segunda etapa del siglo XIX (Tissandier, 1881), y principalmente en tiempos modernos, en un instrumento novedoso y estimulante para despertar la atención del estudiante, catalizar su proceso cognitivo, y hacer más accesible la comprensión de los diferentes principios que gobiernan el funcionamiento de cada uno de ellos (Aref, Hutzler \& Weaire, 2007; Featonby, 2005; Güémez et al., 2009; Jacobsen, 2005; Levinstein, 1982; Miller, 1959; Sarquis \& Sarquis, 2005; Ziegler, 1977).

En el campo específico de la ingeniería, ejemplos de intentos por introducir mediante este enfoque innovadores sistemas instruccionales para estudiantes tanto prospectivos como activos y de diferentes niveles de aprendizaje en diversos aspectos relacionados con ciencia, ingeniería y tecnología han probado la conveniencia de esta herramienta como alternativa diferente, o al menos complementaria, de los tradicionales estilos de enseñanza. Caso concreto es el programa "Joy of Engineering" desarrollado en la pasada década por un grupo multidisciplinario de profesores de diferentes niveles con el propósito de "traer un sentido de diversión y creatividad a los campos de matemática, ciencia e ingeniería" como uno de sus objetivos principales (Phillips et al., 2002, Palazolo et al., 2001). Otros dos ejemplos pueden 
citarse en este punto. El primero lo constituye el programa "Jugando con la tecnología", iniciado en la Universidad del Estado de Iowa en los Estados Unidos durante el periodo académico 1996-1997 (http://www.eng.iastate.edu/twt/). El proyecto tiene como objetivo principal la explicación de los principios subyacentes de muchas de las innovaciones tecnológicas actualmente en uso, mediante la realización de experiencias prácticas de laboratorio con sistemas simples construidos con base en juguetes de una específica marca industrial $\left(\operatorname{LEGOs}^{\circledR}\right)$, y controlados por pequeños microcomputadores. La por lo menos duplicación del número esperado de personas matriculadas en el programa en poco más de una década de funcionamiento, incluyendo profesionales recién egresados, estudiantes de secundaria y universidad, y profesores de estos mismos niveles, habla por sí solo de la acogida de esta herramienta en el mundo académico de su influencia.

El segundo ejemplo tiene que ver con los talleres de enseñanza ASCE ExCEED, realizados como parte de un esfuerzo conjunto del Departamento de Ingeniería Civil y Mecánica de la Academia Militar de Estados Unidos (USMA) y la editorial McGraw-Hill, y cristalizados en la página web http://www.handsonmechanics.com (Welch \& Klosky, 2007). El sitio web provee una serie de entre 25 y 30 modelos físicos y demostraciones probadas, inicialmente para profesores de ingeniería civil, las cuales pueden fácilmente ser implementadas por cualquier instructor, cubriendo las áreas de estática, dinámica, mecánica de materiales, ciencia de los materiales, termodinámica, mecánica de fluidos, transferencia de calor y análisis estructural. Los resultados de la evaluación del programa desde su iniciación en 1999 plantean una enorme aceptación por parte de los estudiantes participantes en términos de un fortalecimiento de su aprendizaje y de una incrementada motivación hacia éste.

Siendo muchos los juguetes y demostraciones asociadas actualmente disponibles para explicar principios científicos de diferente orden, este artículo se enfoca, por razones de espacio, en sólo uno de ellos, conocido por el público en general. La escogencia, si se quiere algo arbitraria, se ha hecho atendiendo principalmente criterios de pertinencia con el objetivo central del artículo, y la relevancia que la ejemplificación de este juguete ha tenido como herramienta pedagógica en la ilustración de algunos conceptos y principios en diversos cursos de programas de pregrado de algunos programas de ingeniería.

\section{Pájaro bebedor}

Este clásico, divertido, y muy conocido juguete científico, inventado hace más de sesenta años por el ingeniero Miles V. Sullivan (Sullivan, 1946), y fabricado desde entonces por diferentes industrias, se constituye en una herramienta de gran potencial pedagógico para la ilustración de una gran variedad de principios en áreas principalmente relacionadas con las ingenierías química y mecánica.

En esencia, la estructura del juguete consta de los siguientes elementos (figura 1a):

Un tubo recto de vidrio, representando el cuerpo del pájaro, unido en cada uno de sus extremos a bulbos del mismo material. El tubo penetra en una pequeña proporción en el bulbo superior en forma similar al cuello de un embudo, y se extiende como un pitillo en una bebida casi que completamente hasta el fondo del bulbo inferior. El bulbo superior, incluyendo el pico de extensión, está recubierto de un fieltro. El único requisito del bulbo inferior, por su parte, es el de no tener ningún tipo de aislamiento que pueda eventualmente afectar el funcionamiento global del juguete. El sombrero es simplemente un accesorio decorativo, mientras que las plumas de la cola contribuyen al balance del pájaro.

Una barra metálica transversal localizada aproximadamente a la altura de la parte media del tubo de vidrio, la cual permite que el aparato oscile o pivotee sobre un soporte (las piernas). La barra puede colocarse estratégicamente de tal manera que el punto de oscilación de localiza ligeramente 
por detrás del cuerpo del tubo, evitando así que el juguete al funcionar caiga hacia atrás.

Un fluido muy poco viscoso y denso, muy volátil, de bajo calor de vaporización, y en una cantidad cuidadosamente medida, llenando parcialmente el tubo del cual previamente se ha evacuado aire. Freón $11\left(\Delta H_{\mathrm{v}}=182.1 \mathrm{~kJ} / \mathrm{kg}\right)$ y diclorometano $\left(\Delta H_{\mathrm{v}}=281 \mathrm{~kJ} / \mathrm{kg}\right)$ son los fluidos principalmente utilizados para este propósito si se los compara, por ejemplo, con agua $\left(\Delta H_{\mathrm{v}}=2257 \mathrm{~kJ} / \mathrm{kg}\right)$, o etanol $\left(\Delta H_{\mathrm{v}}=841 \mathrm{~kJ} / \mathrm{kg}\right)$. Mercurio, con un calor de vaporización de $281 \mathrm{~kJ} / \mathrm{kg}$, parecería ser un fluido apropiado, pero su costo, peligrosidad, e inconveniente densidad obligarían un rediseño del equipo en orden a asegurar un apropiado funcionamiento del juguete.
El comportamiento del juguete puede seguirse con la secuencia real mostrada en la figuras $1 \mathrm{~b}$ y 1c (ver también http://www.youtube.com/watc $\mathrm{h}$ ? $\mathrm{v}=$ nyczz9NsFgs\&feature=related). Se supone que la posición inicial es estática erecta, con el bulbo superior y el pico humedecidos y el nivel de líquido llenando prácticamente el bulbo inferior y la porción baja del tubo conector (figura 1b). Un vaso de agua se coloca a una distancia determinada, de tal forma que el pico, una vez el pájaro alcance posición horizontal, se introduzca en el agua. Si el aire que rodea el juguete no se encuentra saturado de agua, la evaporación del agua de la pared externa del bulbo superior se sucede por dos procesos simultáneos de transferencia de calor y de materia. El agua se convierte en vapor y se integra al aire ambiental a costa de

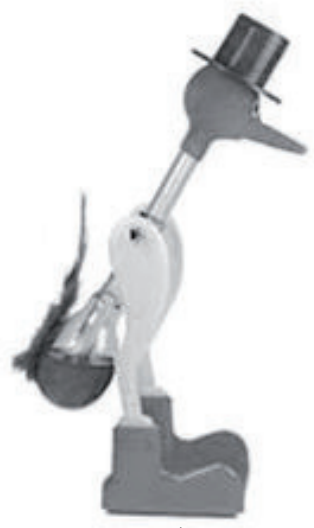

a)

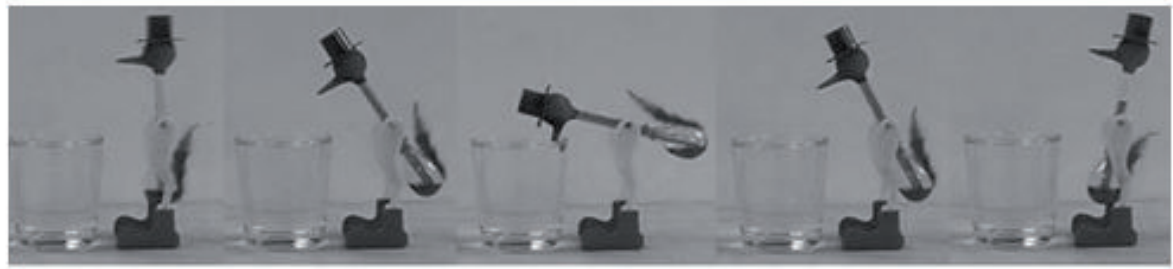

b)

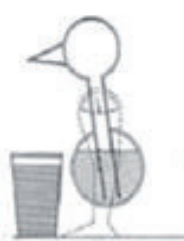

(a)

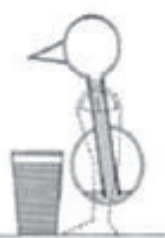

(b)

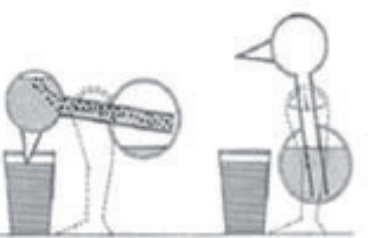

(c)

(d)

c)

Figura 1. a) Pájaro bebedor. b) Secuencia del funcionamiento, c) Secuencia de movimientos 
un enfriamiento de ese bulbo. El descenso de temperatura motiva una condensación parcial del vapor y una disminución de la presión de vapor en el bulbo superior respecto a ésta en el inferior. El gradiente de presión forza el líquido a subir por el interior del tubo al bulbo superior (figura 1c), volviendo este último más pesado, variando su centro de gravedad, y haciendo oscilar el juguete a una posición casi horizontal en la que el extremo inferior del tubo alcanza un nivel superior al de la superficie del líquido (figura 1c). Las presiones en los dos extremos entonces se igualan, los vapores del bulbo superior se mezclan con los provenientes del inferior, y el líquido desciende al bulbo inferior, recuperando el pájaro su posición vertical inicial (figura $1 \mathrm{~b}$ y 1c). La evaporación del agua que moja el bulbo superior se sucede de nuevo y el proceso se repite.

El modelo del pájaro bebedor, hasta donde el autor conoce, no ha sido propuesto, y menos servido, para implementar aplicaciones prácticas en algún área de la ingeniería, salvo alguna más bien anecdótica que se incluye al concluir la presente sección. La importancia del juguete es esencialmente didáctica en la demostración de numerosos conceptos. Algunos resultan obvios pero igualmente ilustrativos, como por ejemplo el de ecuaciones de estado que permite verificar la variación de la presión con cambios en temperatura a volumen constante, tal y como ocurre en el bulbo superior del pájaro; el de presión de vapor y la diferencia entre evaporación y ebullición; el de capilaridad y la absorción de agua en el fieltro que recubre el pico del juguete; ó el de centro de masa que ayuda a explicar el desbalanceo del pájaro. Otros conceptos más complejos, principalmente relacionados con el área de la termodinámica y los procesos de transferencia de calor y de materia, tienen en el funcionamiento del juguete una oportunidad única de ser explicados de manera más práctica y sencilla.

Representación termodinámica: Un rápido vistazo de su funcionamiento puede llevar a pensar en una máquina de movimiento perpetuo debido a la aparente inexistencia de una fuente externa de energía asociada al juguete, más aún si se tiene en cuenta que para un apreciable contenido de agua en el vaso próximo al juguete, este último podría funcionar sin problemas por espacio de varios días. La evaporación del agua con la que el pájaro 'sacia su sed' es ese combustible que funciona a manera de fuente externa de energía para hacer funcionar el juguete y promover la diferencia de temperatura entre los bulbos y el correspondiente flujo de calor. A un nivel más fundamental, la capacidad para producir trabajo en un juguete como éste radica en la diferencia entre los potenciales químicos del líquido externo en el bulbo del pájaro y su vapor insaturado (no en equilibrio). Este 'combustible', sin embargo no es eterno, y aunque $30 \mathrm{~cm}^{3}$ de agua son suficientes para que el pájaro trabaje entre 3 y 4 días en verano, igualmente la sustancia debe ser periódicamente repuesta. Bajo estas condiciones, termodinámicamente hablando, el pájaro es un dispositivo mas de ingeniería, que permite crear movimiento, realizar trabajo mecánico, y por tanto debe ser considerado como un ejemplo de máquina térmica.

\subsection{Ciclo termodinámico}

El juguete puede asemejarse en el lenguaje termodinámico a una máquina de vapor, en donde el fluido principal es precisamente el retirado en forma de vapor de la parte externa del bulbo superior, siendo indispensable para su funcionamiento que su presión parcial sea menor que la de saturación del vapor en el aire ambiente. El ciclo de potencia correspondiente puede configurarse asumiendo por un lado que la temperatura de bulbo superior es aproximadamente la de bulbo húmedo del cuarto, y completándolo suponiendo que el agua evaporada se condensa en la atmósfera a la correspondiente temperatura de rocío. La figura 2 representa el diagrama $T-S$ de esta potencial máquina de vapor.

Si bien el pájaro nunca fue diseñado como un dispositivo de conversión de energía, diversos estudios han intentado determinar cifras termodinámicas asociadas con su funcionamiento. 


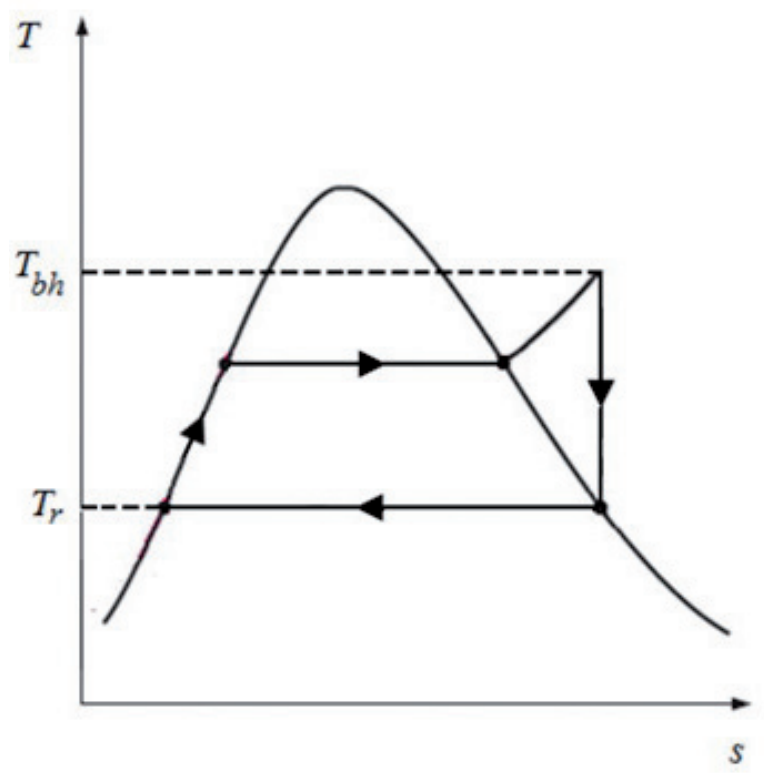

Figura 2. Diagrama T-s del ciclo termodinámico del pájaro bebedor

Uno de ellos es el experimento llevado a cabo bajo condiciones rudimentarias por Don Rathjen, un profesor norteamericano de física, haciendo uso de materiales simple (Rathjen, 1994). Conectando un modelo del pájaro (o pato, como algunos lo identifican) bebedor a un juego de poleas, y haciendo uso de él para levantar livianos clips de papel, según se muestra esquemáticamente en la figura 3, Rathjen logró determinar la magnitud de una nueva unidad de potencia, el 'duckpower' o 'birdpower' (en directa relación con el 'horsepower' asociado con otro animal, el caballo). Actuando como máquina térmica, el pájaro logró levantar el clip una distancia total de $4.4 \mathrm{~cm}$ en un tiempo de 30 minutos, lo que equivale a desarrollar un 'poderoso' nano-caballo de fuerza (equivalente a $1 \mu \mathrm{W}$ ), o, en otras palabras según él mismo concluyó, que " iiise requiere un billón de pájaros para realizar el mismo trabajo de un caballo!!!”.

\subsection{Eficiencia termodinámica}

Estudios más elaborados se relacionan con la eficiencia termodinámica del aparato. Teniendo en cuenta que la cantidad de calor disponible se reparte en este caso entre el trabajo requerido para producir el movimiento del pájaro y el calor necesario para evaporar el agua externa del bulbo superior, pruebas experimentales disponibles lograron determinar una eficiencia del dispositivo de $0.0002(0.02 \%)$, mucho menor que la de por si extremadamente reducida eficiencia ideal de 0.011 (1.1\%), calculada a partir de consideraciones termodinámicas $(\mathrm{Ng} \& \mathrm{Ng}, 1993)$.

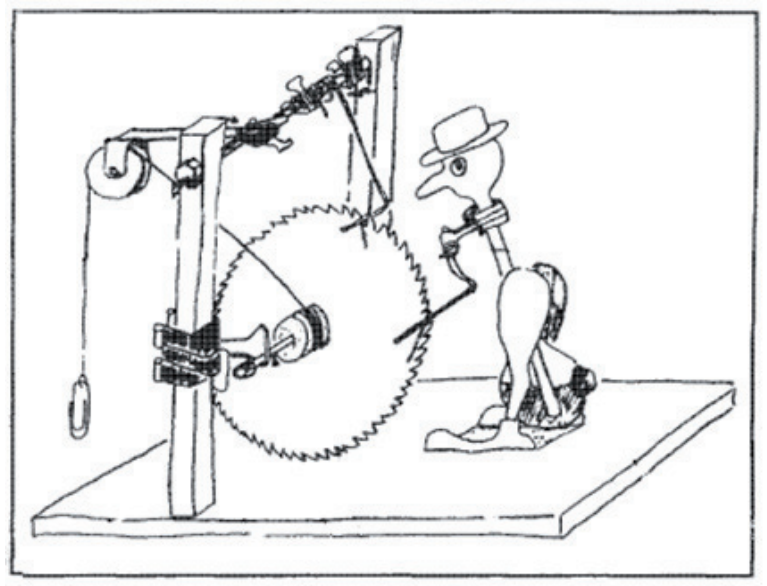

Figura 3. Montaje para la determinación del trabajo mecánico realizado por el pájaro

\subsection{Psicrometría y equilibrio gas vapor}

Estudios cuantitativos han evaluado la influencia de parámetros externos en el período de funcionamiento del pájaro, es decir el tiempo transcurrido entre una inclinación y la siguiente. Los estudios se han enfocado en tres variables principales: la velocidad y humedad relativa del aire ambiente y la naturaleza del compuesto que moja el bulbo superior. Como resulta previsible, la velocidad del viento afecta notablemente la evaporación del agua. Resultados experimentales reportados bajo condiciones de humedad relativa constante $(64 \%)$, mostraron que variaciones de un aire completamente quieto a corrientes con velocidades promedio de $1 \mathrm{~m} / \mathrm{s}$ y $2.5 \mathrm{~m} / \mathrm{s}$, ya sean libres o inducidas por un ventilador, por ejemplo, motivaron disminuciones en el período del orden de 6 y $8 \%$, respectivamente (Murrow, 1966).

En lo que se refiere a humedad relativa, ésta afecta el funcionamiento del pájaro sólo si el líquido 
externo es agua. Si la presión de vapor de agua en el aire es igual a la presión de vapor del agua a la temperatura del lugar (ya sea por condiciones naturales o por humidificación forzada cuando, por ejemplo, se tapa el juguete con una campana, y la continua evaporación hace que se alcance pronto esa condición), el aire exterior estará saturado de agua $\left(H_{\mathrm{R}}=100 \%\right)$, y al no poder llevarse más cantidad de ésta, el proceso de evaporación concluirá, y el movimiento oscilatorio del juguete automáticamente cesará. Si por el contrario, las dos presiones mencionadas son muy diferentes, y por ende la humedad relativa es muy baja, esta diferencia se convierte en una gran fuerza impulsora para la transferencia de materia de fase líquida a vapor, e, indirectamente, para la transferencia de calor necesaria para colocar el agua en la fase requerida, ocasionando de paso una significativa disminución en el período de oscilación del pájaro. Estudios cuantitativos permitieron establecer dos dependencias lineales diferentes entre estas variables, ambas en términos de ley de potencias diferentes, para igual número de rangos de humedad (Güémez et al., 2003).

En lo que se refiere al líquido externo, si este es diferente al agua, su presión parcial en el aire original es seguramente cero, el gradiente impulsor de cambio es siempre grande, y el periodo de oscilación variará en una medida que depende del calor de vaporización de la sustancia en cuestión. Es así, por ejemplo, que si se utiliza etanol $\left(\Delta H_{\mathrm{v}}=841 \mathrm{~kJ} / \mathrm{kg}\right)$, el periodo de oscilación disminuirá sensiblemente -alrededor de cinco veces respecto del caso usando agua- (y no exactamente por el alcoholismo del pájaro !!!), pero de cualquier manera menos que si se trata de acetona $\left(\Delta H_{\mathrm{v}}=551.7 \mathrm{~kJ} / \mathrm{kg}\right)$, por ejemplo. Estudios no concluyentes indican así mismo una interesante sensibilidad del periodo con la temperatura ambiente, de tal manera que si esta última se incrementa el periodo disminuye (Lorenz, 2006).

\subsection{Transferencia de calor}

Ya se observó que el gradiente de temperatura entre los dos bulbos, debido a la evaporación del agua exterior, es el elemento responsable del movimiento del juguete. Una modificación que implica eliminar el agua exterior del diseño original brinda una posibilidad de incorporar nuevos conceptos en la descripción del movimiento. En la nueva versión se elimina el vaso de agua, el pico del pájaro se mantiene por ende seco en todo momento, y ningún tipo de evaporación se sucede. El gradiente de temperatura requerido para el funcionamiento del juguete se fabrica entonces artificialmente al pintar el bulbo inferior de color negro y colocarlo en las proximidades de una fuente de calor, ya sea una lámpara caliente o la misma luz solar, mientras que el bulbo superior se mantiene a la sombra (o se pinta igualmente, pero con una pintura reflectiva color plata o blanco). La absorción del calor radiante eleva la temperatura del bulbo inferior, mientras que la reflexión del mismo en el bulbo superior mantiene la temperatura más baja de este último (Güémez et al., 2004). Este 'pájaro solar' no sólo puede funcionar sin agua, sino que lo hace de manera independiente de las condiciones climáticas ambientales (humedad relativa) que, como antes se mencionó, limitaban el movimiento en la versión original del juguete.

Otra modificación de la versión original, igualmente sin agua, implica atar un pedazo de hielo al bulbo superior, y así crear el gradiente mencionado (Ford \& Brittain, 1997). La fusión del hielo y el enfriamiento por evaporación del líquido residual son los fenómenos que en este caso se suceden, permitiendo la extracción de calor de un proceso endotérmico espontáneo. Pruebas experimentales indican que esta versión funciona de manera más uniforme que la original, incluso a humedades relativas altas.

Una historia real alrededor de este juguete, y su posibilidad de convertir energía térmica en un diferencial de presión al interior del aparato y realizar trabajo mecánico, se relaciona con una propuesta de utilizarlo potencialmente a gran escala como fuente de energía, así esta última sea de un orden de magnitud muy pequeña, y así hacer más útil y productivo el empleo de la fuerza 
laboral agrícola en ambientes primitivos típicos de países subdesarrollados (Murrow, 1966). En 1966, y como consecuencia de un corto viaje de turismo a Egipto, el ingeniero aeronáutico americano R.B. Murrow planteó la construcción de un gigante pájaro bebedor como una alternativa factible a la extracción de agua de canales, u otras fuentes disponibles para la irrigación de terrenos en zonas bajas. En ausencia de equipos de tecnología moderna, esta última actividad era, y probablemente aun es, realizada con ayuda de obsoletos elementos tales como tornillos, norias y cigoñales, entre otros, con los correspondientes elevados consumos de energía humana o animal. El análisis teórico inicial fue seguido primeramente por series de pruebas experimentales a pequeña escala con prototipos cuyos modelos habían sido debida y convenientemente adaptados a las condiciones climáticas del lugar respecto del modelo original. La etapa final incluyó discusión de las posibilidades y condiciones de posible escalamiento del modelo. Si bien el resultado del estudio preliminar permitió a Murrow establecer la factibilidad técnica de la alternativa, las cifras acompañantes, teniendo en cuenta sólo criterios crudamente cuantitativos y no de beneficio social, resultaban poco atractivas. Bajo criterios económicos de la época, se requeriría el funcionamiento 24 horas al día de cuatro unidades de versión veinte veces más grande que el modelo original del juguete para realizar el mismo trabajo que un hombre promedio haría en ocho horas. Si el escalamiento fuera de treinta veces el tamaño original, el número de pájaros bebedores gigantes requerido disminuiría a tres. Escalamientos mayores implicarían dificultades asociadas con la portabilidad de los pájaros (el autor desconoce si algún pájaro bebedor gigante actualmente está siendo efectivamente utilizado para bombear agua en tierras de Egipto).

\section{Conclusiones}

Los juguetes no son sólo cosa de niños. Una apropiada selección de ellos permite visualizar de manera clara y sencilla, haciendo y no viendo hacer, una variedad de conceptos y principios importantes que de otra manera serían más difíciles de entender. La integración de estos conceptos en ciencia, ingeniería y tecnología, alrededor del funcionamiento de un buen número de juguetes, unida a la fácil disponibilidad de estos últimos, relativo bajo precio, y posibilidad de utilización en lugar de aparatos estándar de laboratorio, hace supremamente valiosa su incorporación en procesos de enseñanza a éste y otros niveles académicos.

La comparación de hombres de ciencia con infantes, sin embargo, si bien suena graciosa y hasta un poco exagerada, no resulta de plano completamente descabellada en este contexto. Historiadores e investigadores han hecho referencia al hecho de "los científicos son como niños" (Porter, 1988; Valsineer \& van der Veer, 2000), y como tal "personas que nunca pudieron desprenderse de sus juguetes" (Laithwaite, 1971). Hechos aislados asociados con reconocidos hombres de ciencia se constituyen en ejemplos de esta afirmación. Una famosa fotografía (figura 4), tomada durante la inauguración del Instituto de Física en la Universidad de Lund, Suecia, el 31 de Mayo de 1951, muestra a dos Premios Nobel, el Danés Niels Bohr (18851962) y su discípulo, el austriaco Wolfgang Ernst Pauli (1900-1958), observando con interés el extraño comportamiento, hasta ese momento no completamente entendido, del llamado tippe tap o trompo mágico. Del físico Alemán Ludwig Prandtl (1875-1953), pionero contribuidor en temas de aerodinámica, se dice que no podía resistir la tentación de manipular y experimentar con cualquier juguete que se encontrara, a tal punto que abandonaba temporalmente cualquier tipo de evento en que estuviera participando para hacerlo (Vogel-Prandtl, 2004). El primer tratado sistemático sobre la física del billar, publicado en 1835, es debido al ingeniero y matemático Francés Gaspard-Gustave Coriolis (1792-1843), ampliamente conocido por la fuerza y el efecto en dinámica de fluidos que llevan su nombre, siendo ésta, sin embargo, una de sus obras menos conocidas (Coriolis, 1835). 
El matemático alemán Christian Felix Klein (1849-1925) y su compatriota, el físico alemán Arnold Sommerfeld (1868-1951), invirtieron alrededor de década y media en la escritura de una obra en cuatro volúmenes relacionada con las teorías físico-matemáticas del giroscopio (Klein \& Sommerfeld, 1897-1910).

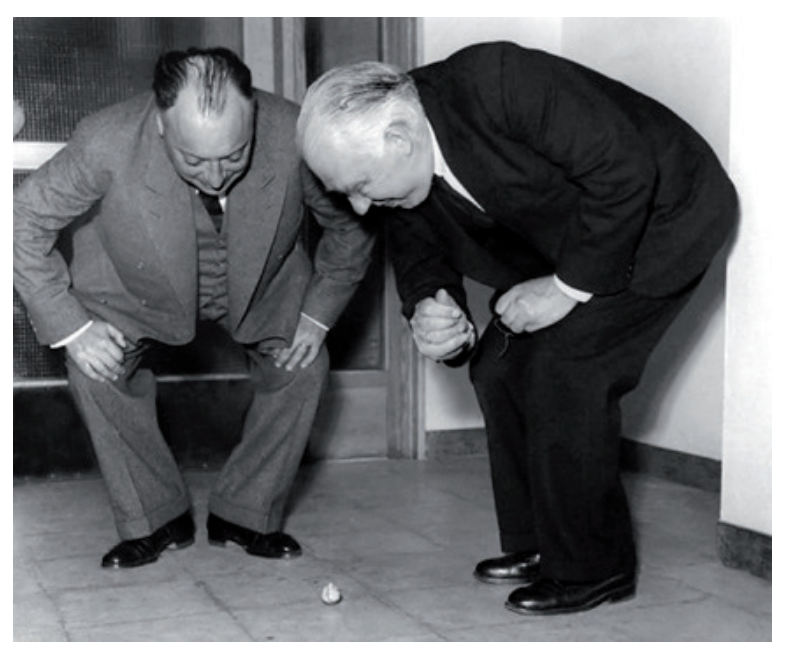

Figura 4. Niels Bohr (derecha) y Wofgang Pauli (izquierda) observando el funcionamiento del tippe top. Credit: Fotografía por Erik Gustafson.

Cortesia: Fysiska institutionen, Lunds Universitet.

Los juguetes permiten el modelamiento simple de sistemas complejos mientras se preservan, al menos, unos detalles claves de los sistemas originales. $\mathrm{Su}$ poder y aplicabilidad no puede ni debe subestimarse. Su utilización mediante modelos simples, y en la mayoría de veces idealizados, en la introducción de un tópico, el desarrollo cualitativo o cuantitativo de diferentes conceptos, la explicación de sistemas complejos, y la facilitación del entendimiento por parte de los estudiantes, hacen de ellos un muy valioso instrumento pedagógico. De esta manera, es como si los juguetes hubiesen sido creados para la investigación en el salón de clases. La tendencia de algunos profesores a visualizar ciencia en prácticamente cualquier juguete con el que se encuentran, permite que los estudiantes no solo se motiven más, sino que también puedan apreciar el grado de relevancia de la ciencia en todo lo que les rodea en su vida diaria. En el caso de estudiantes más avanzados, la introducción de juguetes se constituye en un medio para desarrollar proyectos de investigación que mediante su construcción en el laboratorio les provee una oportunidad adicional no sólo de aprender, sino de darse cuenta de que la realidad es más rica que la teoría que aparece en los libros.

\section{Referencias bibliográficas}

Al-Gailani, S. (2009). Magic, science and masculinity: marketing toy chemistry sets. Studies in History and Philosophy of Science 40 (4), 372381.

Aref, H., Hutzler, S., \& Weaire, D. (2007). Toying with physics. Europhysics News 38 (3), 23-26.

Brenni, P. (2012). The evolution of teaching instruments and their use between 1800 and 1930. Science \& Education 21 (2), 191-226.

Coriolis, G. (1835). Théorie mathématique des effets du jeu de billard. Paris: Carilian-Goeury.

Featonby, D. (2005). Toys and physics. Physics Education 40 (6), 537-543.

Ford, D.I., \& Brittain, S.D. (1997). The drinking bird toy and its modifications as a means of illustrating thermodynamic concepts. The Chemical Educator 2 (5), 1-6.

Güémez, J., Fiolhais, C., \& Fiolhais, M. (2009). Toys in physics: lectures and demonstrations - a brief review. Physics Education 44 (1), 53-64.

Güémez, J., Valiente, R., Fiolhais, C., \& Fiolhais, M. (2004). A big sunbird. The Physics Teacher 42 (5), 307-309.

Güémez, J., Valiente, R., Fiolhais, C., \& Fiolhais, M. (2003). Experiments with the drinking bird. American Journal of Physics 71 (12), 1257-1263.

Jacobsen, E.K.(2005). JCE resources for chemistry and toys. Journal of Chemical Education 82 (10), 1443-1446. 
Klein, F., Sommerfeld, A. (1897-1910). Über die Theorie des Kreisels (4 vols.). Leipzig: B.G. Teubner.

Laithwaite, E. (1971). Scientific toys. New Scientist 52, p. 233.

Levinstein, H.(1982). The physics of toys. The Physics Teacher 20 (6), 358-365.

Lorenz, R. (2006). Finite-time thermodynamics of an instrumented drinking bird toy. American Journal of Physics 74 (8), 677-682.

Miller, J.S. (1959). Toys and physics. School Science and Mathematics 59 (5), 359-370.

Murrow, R. B. (1966). A simple heat engine of possible utility in primitive environments. The Rand Corporation paper P-3367, Santa Monica, California.

Ng, L.M., \& Ng, Y.S. (1993). The thermodynamics of the drinking bird toy. Physics Education 28 (5), 320-324.

Palazolo, P., Phillips, A., Camp, C., (2001). Toys, Tinkerers, and Tomorrow: Growing Engineers. Proceedings of the 2001 American Association of Engineering Education (ASEE) Annual Conference \& Exposition, Albuquerque, The United States, Session 3453.

Philips, A.P., Palazolo, P.J., Magun-Jackson, S., Camp, Ch. V., \& Schmucker, D. (2002). Powerful Play: Using Toys as Tools in Engineering Education. Proceedings of the 2002 American Society for Engineering Education (ASEE) Annual Conference \& Exposition, Montreal, Canada, Session 1615.
Porter, R.S. (1988). Man Masters Nature: Twentyfive centuries of science. New York: G. Braziller.

Rathjen, D. (1994). Duckpower. Exploring 18 (4), 7-8.

Sarquis, J.L., \& Sarquis, A.M. (2005). Toys in the classroom. Journal of Chemical Education 82 (10), 1450-1453.

Sullivan, M.V., 1946. Novelty device. USPat. 2402463, Junio 18.

Tissandier, G. (1881). Les Récréations Scientifiques ou l'Enseignement par les jeux, 3a ed.. Paris: Masson.

Turner, R. (1992). 100 years of physics and toys: Balancing toys. The Physics Teacher 30 (9), 542543.

Valsineer, J., \& van der Veer, R. (2000). The Social Mind: Construction of the Idea. Cambridge: Cambridge University Press.

Vogel-Prandtl, J. (2004). Ludwig Prandtl: a biographical sketch, remembrances and documents. Trieste: International Centre for Theoretical Physics.

Welch, R.W., \& Klosky, J.L. (2007). An online database and user community for physical models in the engineering classroom. Advances in Engineering Education 1 (1). p. 1-25.

Ziegler, G.R. (1977). Toys in the chemistry classroom. Journal of Chemical Education 54 (10), p. 629. 